\title{
La competencia evaluativa de los docentes: formación, dominio y puesta en práctica en el aula
}

\author{
The assessment competence of teachers: training, expertise and \\ classroom implementation
}

\author{
Aida Sanahuja Ribés \\ Lucía Sánchez-Tarazaga Vicente \\ Universitat Jaume I (UJI), España
}

\section{Resumen}

Hoy en día es necesario que los docentes demuestren la competencia evaluativa: saber evaluar, saber cómo evaluar y poner en práctica la evaluación con un amplio conjunto de técnicas y métodos. En este trabajo se muestran los resultados de una investigación cuyos objetivos han sido: 1) conocer si los docentes se han formado o han leído sobre diferentes técnicas y métodos de evaluación; 2) averiguar el nivel de dominio que tienen de las diferentes técnicas y métodos de evaluación; 3) comprobar si han puesto en práctica dichas técnicas y métodos en el aula. El diseño metodológico es de tipo descriptivo, basado en el método de encuesta y a cuyo cuestionario ha respondido una muestra de 193 docentes españoles. Los resultados apuntan que la autoevaluación es el método de evaluación sobre el cual más se han formado los docentes y el más utilizado en el aula. Por contra, el cuestionario KPSI es el instrumento sobre el que menos se han formado y por ende, el menos empleado en el aula. Una de las principales conclusiones que se derivan del estudio gira en torno a la importancia de mejorar la formación del profesorado para potenciar el dominio de la función evaluadora de este colectivo.

Palabras clave: competencia evaluativa; formación docente; técnicas de evaluación; métodos de evaluación.

\begin{abstract}
Nowadays it is necessary that teachers demonstrate the assessment competence: know about assessment, know how to assess and put into practice the assessment with a wide set of techniques and methods. This paper reports the findings from a study that aimed to analyse: 1) know if teachers have been trained or have read about different assessment techniques and methods; 2) find out the level of expertise they have of the different assessment techniques and methods; 3) check if they have put these techniques and methods into practice in the classroom. The methodological design is descriptive and survey-based, whose questionnaire was answered by a sample of 193 Spanish teachers. The results suggest that the self-assessment is the evaluation method on which most teachers have been trained and the most used in the classroom. On the other hand, the KPSI questionnaire is the instrument on which less have been formed and therefore, the least used in the classroom. One of the main conclusions that derive from the study revolves around the importance of improving teacher training to enhance the assessment function of this collective.
\end{abstract}

Keywords: assessment competence; teacher training; assessment techniques; assessment methods. 


\section{INTRODUCCIÓN}

Dado que evaluar es emitir un juicio sobre el valor de una cosa, saber evaluar se convierte en un asunto complejo y delicado... que nos exige una actuación profesional seria y formada, reflexiva, deliberada, intencional, sistemática y, sobre todo, que se pueda justificar; ... y es que junto al 'qué' evaluar, al 'para qué' y al 'cómo', hay un 'quién', el que evalúa, y unos 'para quién' que son los destinatarios de esa actividad (Trillo Alonso, 2005).

Partimos de la convicción junto con Sanmartí (2007) de que la evaluación es el motor del aprendizaje, es fundamental en tanto que orienta la actividad educativa. Además, su relevancia ha crecido en las últimas décadas motivado, en parte, por la preocupación acerca de la mayoría de sistemas educativos de países avanzados por el rendimiento académico y su evaluación. Como muestra, en España, contamos con los informes que realiza el Instituto Nacional de Evaluación Educativa (INEE, anterior INCE), mientras que en la escala europea, nos referimos a los más que conocidos informes PISA (coordinado por la OCDE) donde pretenden evaluar las competencias del alumnado de secundaria obligatoria y las pruebas TIMMS y PIRLS (promovido desde la IEA, en inglés: International Association for the Evaluation of Educational Achievement) para evaluar al alumnado de primaria.

Vivimos en una sociedad que tiene escasa relación con la de hace unas décadas. Los acelerados y constantes cambios (a nivel cultural, social y pedagógico) que se han producido han modificado la realidad de las aulas y, por ende, el trabajo de sus docentes, incrementando la complejidad en las funciones que deben desempeñar (Prats, 2016), entre ellas la de evaluar. La globalización, la inmersión en la era digital, el aprendizaje a lo largo de la vida y la extensión de los espacios de aprendizaje, la escolarización generalizada de todas las niñas y niños de educación primaria, la integración educativa de la diversidad así como la orientación del currículum para desarrollar las competencias clave del alumnado son algunas de las cuestiones que desafían el papel tradicional que ha ocupado la evaluación.

En este contexto, y siguiendo con Murillo e Hidalgo (2015), es indispensable una evaluación que promueva la participación y transformación social para conseguir una sociedad con valores impregnados de equidad y respeto. Una evaluación continua y procesual para el desarrollo integral del discente, que sea a la vez crítica, reflexiva y democrática. Además, dado que el proceso de recogida de la información es complejo y lleno de matices esto implica, a su vez, conseguir una rica variedad de evidencias. Para ello, el docente debe, además, conocer y saber 
utilizar diferentes instrumentos y técnicas de evaluación, con el fin de garantizar un proceso válido (Cohen, Manion y Morrison, 2000; Darr, 2005), confiable (Cohen y Swerdlik, 2009) y lo más imparcial posible o sesgo (Covacevich, 2014). Es lo que denomina Tejada (2010) como el principio de "multivariedad y triangulación instrumental" (p.731), imprescindible para recopilar evidencias de aprendizaje de las competencias del discente.

Por ello tanto expertos en este campo como los organismos internacionales reconocen la evaluación como una de las competencias necesarias en la concepción del perfil del docente del siglo XXI, tal y como recoge un trabajo de Sánchez-Tarazaga (2017). Así, diferentes autores (Caena, 2014; Cano, 2005; Galvis, 2007; Perrenoud, 2004; Sarramona, 2007; Tiana, 2013) consideran que la evaluación para el aprendizaje debe formar parte del marco competencial del profesorado. En cuanto a los organismos internacionales, por un lado encontramos los que identifican de forma explícita la competencia evaluativa a través de diferentes parámetros, como, por ejemplo, una necesidad de "integrar la evaluación sumativa y formativa" (OCDE, 2009) o "evaluar el avance en el aprendizaje y el grado de adquisición de las competencias de los alumnos en la materia que deben aprender" (Banco Mundial, 2007). Por otro, las que tienen en cuenta la evaluación, de forma más implícita, como cualidad del profesorado (FIER, 2010; UNESCO, 2015).

Sin embargo, a menudo los docentes estamos más preocupados por transmitir los contenidos que por verificar si realmente el alumnado lo ha procesado correctamente. Dicho de otro modo, si evaluamos mal, nuestros alumnos aprenderán mal aunque nuestra enseñanza sea 'supuestamente' excelente (Morales, 2009, p.7). $Y$ es que enseñar no es equivalente a aprender y en este punto juega un papel decisivo la competencia evaluativa del profesorado.

La OCDE (2005) identifica los obstáculos que dificultan el cambio de las prácticas de evaluación del profesorado. Además de problemas logísticos (elevadas ratios), los profesores hallan complicado cambiar sus prácticas porque implica cambiar muchos aspectos, como la interacción con el alumnado, la planificación, la atención al alumnado y hasta el modo de concebir el éxito de estos (Martínez Rizo, 2013). Pero además, tal y como más adelante expondremos, por la falta de formación en la competencia evaluativa. 
Teniendo en cuenta las consideraciones arriba planteadas, las preguntas que a continuación nos surgen son: ¿se han formado los docentes en diferentes instrumentos para evaluar? ¿qué grado de dominio tienen sobre los mismos? Y un paso más, ¿lo han puesto en práctica en el aula? Estas preguntas constituyen los ejes temáticos de este trabajo.

\subsection{Cuestiones previas en torno a la evaluación}

a) Finalidades de la evaluación: sumativa, formativa y formadora

El término evaluación, si bien está ampliamente extendido en el vocabulario del colectivo docente, está sujeto en realidad a una importante polisemia, por lo que conviene delimitar su significado. Tal y como reconocen Jorba y Sanmartí (1996), se trata de un proceso en el que se distinguen tres etapas: recogida de información, análisis de la misma y juicio sobre el resultado de este análisis y toma de decisiones de acuerdo con el juicio emitido. Estamos, ante un proceso complejo, que combina una dimensión cognitiva (en la que se construyen conocimientos), instrumental (implica diseñar y aplicar procedimientos e instrumentos) y axiológica (establecer el valor de algo) (Tejedor y García-Valcárcel, 2010).

98 Nos detenemos en la tercera fase arriba señalada pues es en la toma de decisiones donde reside las diferencias en las finalidades de la evaluación. Siguiendo a Sanmartí y Alimenti (1998), si la decisión es de carácter social, la evaluación se convierte en lo que llamamos calificación o evaluación sumativa y tiene como objetivo certificar el nivel de progreso del alumnado al finalizar un periodo de aprendizaje (unidad, trimestre o curso académico). Sin embargo, las decisiones de carácter pedagógico, guardan relación con la denominada evaluación formativa y pretenden identificar los cambios que se han de introducir en el aprendizaje, de modo que el alumnado sea capaz de regular la construcción de conocimiento. Pero además, se ha avanzado en esta evaluación formativa tradicional hacia una de carácter formadora, en la que se empodera al estudiante, de forma que toma un papel activo en su aprendizaje y se le reconoce la capacidad para corregir sus propios errores y tomar decisiones de cambio oportunas.

\section{b) Coevaluación y autoevaluación: hacia la evaluación democrática}

En el contexto de la evaluación formativa y formadora parece que el aula se convierte en un espacio en el que el alumnado sea capaz de autorregularse. En este sentido, la siguiente cuestión que pretendemos abordar es el 'quién' debe liderar la función evaluativa. 
Sanmartí (2007) explica que solo quién ha cometido los errores es capaz de corregirlos. Por ello, el docente ha de ser consciente de esta premisa y articular mecanismos para conseguir la participación del discente en la evaluación. Tal y como afirma López (2005), los educadores deben fomentar la participación del alumnado en los procesos de evaluación a partir de la autoevaluación (que el alumno se evalúe a él mismo), coevaluación (evaluación entre pares) y evaluación compartida (diálogo entre alumnado y docente sobre la evaluación del aprendizaje).

López, González y Barba (2006) van más allá y hablan de la evaluación democrática, la cual presenta las siguientes características: intercambio de información; participación del alumnado en el proceso; uso de estrategias para la negociación y cogestión del currículum; comunicación y respeto entre docente y alumnado; progresar hacia procesos de autocalificación (calificación dialogada) y realizar una metaevaluación.

En esta misma línea, Murillo e Hidalgo (2016), también se posicionan a favor de la evaluación democrática de los alumnos. En concreto, la caracterizan a partir de los siguientes axiomas: 1) empoderar a los alumnos en su propio proceso de aprendizaje, 2) pasar de la evaluación de los alumnos a la evaluación con los alumnos (estrategias de autoevaluación que fomenten la reflexión personal), 3) que el alumnado reflexione críticamente y aporte sus puntos de vista, 4) la justicia (dar más a los que más lo necesitan y utilizar estrategias variadas), 5) no ser jerárquica (docente como facilitador), 6) cooperativa (debiendo de ser extendida a la familia) y 7) ser social (evaluación transformadora).

Una modalidad dentro de la coevaluación podría ser la que se utiliza a través de la evaluación del trabajo grupal mediante la metodología de aprendizaje cooperativo. Naranjo y Jiménez (2015) establecen que se trata de una evaluación reguladora y formativa, a través de la cual los equipos van reflexionando y "modelando" su propio funcionamiento, consolidando lo que hacen bien y proponiéndose objetivos de mejora. Éstas proponen que en el Programa CA/AC ("Cooperar para Aprender/ Aprender a Cooperar") coordinado por Pujolàs y Lago, la evaluación del "trabajo en equipo" contempla: una valoración de las diferentes contribuciones de los integrantes del equipo en cuanto a su buena gestión y funcionamiento, una evaluación independiente de la del equipo (p.ej. un equipo con una valoración baja en cuanto a su funcionamiento como equipo, la contribución de uno de sus miembros es alta) y una evaluación individual (calificación). 
Siguiendo a Jiménez y Naranjo (2014), para los docentes el hecho de que los alumnos tomen parte de su propio aprendizaje y tengan responsabilidades en el mismo es importante. Aunque, estas afirman que, en el ámbito B del programa del aprendizaje cooperativo (el trabajo en equipo como recurso para enseñar), sólo el 2\% de los maestros utiliza estructuras cooperativas con el propósito de evaluar. Del mismo estudio se extrae que el $81 \%$ del profesorado utiliza estructuras cooperativas al inicio de la unidad didáctica para extraer los conocimientos previos de los alumnos, pero no para ajustar el producto al programa didáctico. Y al final del proceso, el 19\% utiliza estructuras cooperativas con el objetivo de evaluar.

\section{c) La evaluación auténtica}

Un tercer elemento que debemos introducir es, haciendo referencia al trabajo de Monereo (2009), la "autenticidad" de la evaluación (y la enseñanza), concebida como su vinculación a la realidad del alumnado:

un conjunto de actividades instruccionales y de evaluación, secuenciadas y conectadas en el tiempo, que plantean al alumnado problemas y conflictos a resolver cuyas condiciones contextuales (recursos y limitaciones, exigencia cognitiva, presión emocional, etc.), guardan una gran fidelidad con las condiciones contextuales que los alumnos deberán afrontar en un futuro próximo, en calidad de profesionales del asesoramiento psicoeducativo (Monereo, Sànchez-Busqués y Suñé, 2012, p.81).

Se trata de un tipo de evaluación alternativa (Fortea, Sánchez-Tarazaga, ZorrillaSilvestre, 2017), que debe cumplir una serie de condiciones: realista (simular al máximo posible los recursos, equipamientos, espacios... en que se circunscribe el proyecto o problema); relevante (los aprendizajes deben tener sentido y la competencia desarrollada debe ser de utilidad al alumnado); constructivista (plantear actividades para captar el proceso de construcción que desarrolla el alumnado con el propósito de ofrecer ayudas ajustadas a los problemas que puedan ir apareciendo); socializante (apropiación de los discursos, signos o valores de identidad de la comunidad profesional en la que el alumnado va a integrarse) (Monereo, Sànchez-Busqués y Suñé, 2012).

\section{d) Herramientas para recabar evidencias: los instrumentos de evaluación}

Si pretendemos llevar a cabo un currículum orientado al desarrollo de las competencias clave del alumnado y que atienda la heterogeneidad presente en el aula, es imprescindible evaluar para regular los aprendizajes y evaluar para comprobar que se ha aprendido (Sanmartí, 2010). Para ello, el docente debe contar con un amplio dispositivo instrumental. Pasamos a presentar algunos de los instrumentos 
utilizados para la evaluación del aprendizaje del alumnado; dado que son conceptos que forman parte del cuestionario aplicado en esta investigación. Estos son: check list (registrar una muestra de las conductas planificadas que se pretende observar), registro de incidentes críticos (cuando la anécdota narra varios comportamientos observados en forma de secuencia), diario (según Bisquerra (2007) ofrece un registro descriptivo y reflexivo de los fenómenos y las respuestas personales ante ellos), portafolio (carpeta que reúne el conjunto de tareas efectuadas a lo largo de todo el proceso de enseñanza- aprendizaje. Tejada (2010) apunta que favorece la autonomía, la reflexión y la autorregulación), presentaciones (defensa oral y comunicación de las producciones realizadas), entrevista (Massot, Dorio y Sabariego (2012) indican que tiene como objetivo obtener información de forma oral sobre acontecimientos vividos y aspectos subjetivos de los informantes), cuestionario KPSI (cuestionario de autoevaluación (diseñado por Tamir y Lunetta, 1978) con la finalidad de que el alumnado valore el grado de dominio sobre un tema (Sanmartí, 2010).

Tal y como explican Arellano, Jara, Merino et al. (2008), se le pide al alumnado que elija su respuesta en función de unos niveles), rúbrica (guías de puntuación que describen o presentan los criterios, los parámetros o las características específicas de una tarea en varios niveles de rendimiento (Martínez-Rojas, 2008), con el fin de clarificar lo que se espera del trabajo y de facilitar la correspondiente retroalimentación (Tejada, 2010). El resultado se materializa en una matriz), examen escrito (la RAE lo define como "prueba (escrita u oral) que se realiza para demostrar la suficiencia en una materia determinada o la aptitud para cierta actividad o cargo") y contrato de aprendizaje (De Miguel (2005) lo define como un pacto concreto entre el docente y el alumno para la obtención de unos aprendizajes a partir de una propuesta de trabajo de carácter autónomo, con una supervisión del docente y durante un periodo acordado. Fernández March (2006) recogen algunas ventajas como la promoción del trabajo autónomo y responsable del alumnado y la atención a la diversidad, intereses y ritmos).

\subsection{Lo que entienden los docentes por evaluación}

Pero, ¿qué entienden los docentes por evaluación? En la obra Evaluación Educativa: una aproximación conceptual, Elola y Toranzos (2000), recogen las representaciones más usuales que tienen los maestros en relación a la evaluación. Estas son: a) la asocian a los exámenes, b) sirve para emitir juicios de valor sobre los alumnos, c) se relaciona la nota como un instrumento de control disciplinario, d) encuentran un desfase entre la teoría y la práctica, e) identifican evaluar con calificar, f) se centran 
en la memorización y g) las connotaciones más frecuentes de la evaluación son: control externo, función penalizadora, calcular el valor de una cosa, calificación y grado de suficiencia o insuficiencia de determinados aspectos.

Si buscamos las causas de esta percepción, Stiggings (2007, citado en Martínez Rizo, 2013) afirma que en Estados Unidos la preparación del profesorado en materia de evaluación no ha sido suficiente:

El principal desafío que enfrentamos actualmente en las escuelas en lo relativo a evaluación es asegurar que las prácticas sanas lleguen a cada aula, que las evaluaciones se usen para beneficiar a los alumnos... Este reto ha permanecido sin ser atendido por décadas y parece que ha llegado el tiempo de conquistar esa última frontera de la evaluación: el uso efectivo de evaluación formativa para apoyar el aprendizaje (p.10).

Podemos constatar que en España sucede algo similar. Los programas de formación inicial no aseguran la competencia evaluativa. La formación inicial del profesorado ha sido particularmente débil en lo que se refiere a la evaluación y los planes de actualización o formación permanente que se ofrecen al profesorado en activo. Una de las principales causas de este problema se debe, según Palacios y López-Pastor (2011) a la falta de preparación de los encargados de la acción formativa de los futuros docentes (principalmente al profesorado universitario). Según estos autores, los formadores universitarios perciben que no se encuentran bien preparados para realizar una evaluación formativa y desconocen los beneficios de la misma (Tejedor, 1998, Flórez, 1999), motivado en parte por la falta de formación didáctica específica en cuestiones de evaluación. Esto conduce a que sigan prevaleciendo los modelos tradicionales de evaluación en las aulas preuniversitarias, un hecho preocupante especialmente en los programas de formación inicial del profesorado (tanto a nivel de grado como de máster), pues los estudiantes suelen heredar la cultura profesional de sus docentes (Sánchez-Tarazaga, 2017) y tienden a reproducir en sus clases los métodos evaluativos que han vivido como alumnos.

\section{OBJETIVOS DE LA INVESTIGACIÓN}

Los principales objetivos que guían este trabajo son:

1. Conocer si los docentes se han formado o han leído sobre diferentes técnicas y métodos de evaluación.

2. Averiguar el nivel de dominio que tienen los docentes en referencia a las diferentes técnicas y métodos de evaluación. 
3. Comprobar si los docentes han puesto en práctica las diferentes técnicas y métodos de evaluación en el aula.

Están fundamentados en cómo está articulado el aprendizaje de una competencia, en este caso, la evaluativa y más concretamente en lo que respecta a instrumentos y técnicas de evaluación. Para ello, nos basamos en una aproximación (simplificada) de la pirámide de Miller (1990), según la cual estableció un esquema de evaluación de la competencia profesional organizada en los siguientes niveles, relacionados con los objetivos (ver Figura 1).

\section{FIGURA 1}

\section{Pirámide de Miller (1990), con adaptación de Bernal y Teixidó (2012)}

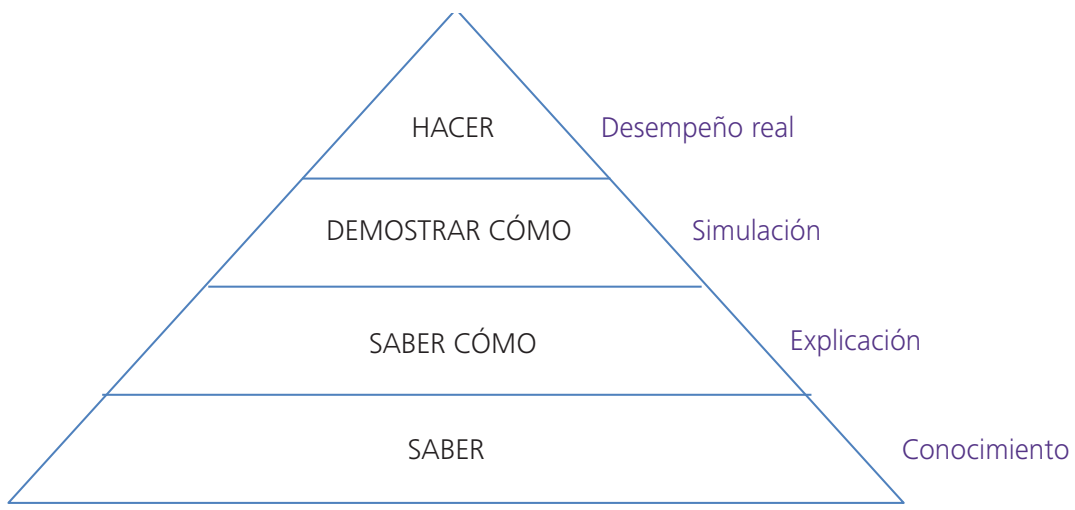

Referido al saber, es decir, los conocimientos, que se encuentran en la base de la misma, corresponde con el objetivo 1 de nuestra investigación, pues se trata de averiguar lo que los docentes saben de evaluación. El siguiente escalón es el de saber cómo realizar la evaluación, esto es, su grado de dominio percibido para esta función, que tiene que ver con el objetivo 2 de estudio. Por razones de simplificación, nos dirigimos directamente al último escalón donde se sitúa el hacer, pues pretendemos estudiar el desempeño real de la función evaluativa de los docentes. Estamos, por tanto, en el objetivo 3.

\section{MÉTODO}

\subsection{Muestra}

En este estudio se empleó el método de muestreo fortuito, dado que se seleccionaron directamente los sujetos de la investigación. Esto es, se tomaron los docentes ya titulados en Magisterio en Educación Infantil y Educación Primaria. De este modo la 
muestra quedó compuesta por un total de 193 informantes, más específicamente $176(91,19 \%)$ eran mujeres y 17 (8,81\%) hombres. A continuación se presenta, en la Tabla 1, la distribución de la muestra según el sexo, la edad, si trabajan o no, años de experiencia y la procedencia de los participantes.

TABLA 1

Distribución de la muestra por sexo, la edad, si trabajan o no, años de experiencia y la procedencia de los participantes

\begin{tabular}{|c|c|c|c|c|c|c|c|c|c|c|c|c|c|c|}
\hline \multicolumn{3}{|c|}{ Sexo } & \multicolumn{3}{|c|}{ Edad } & \multicolumn{3}{|c|}{$\begin{array}{l}\text { Trabajo en } \\
\text { educación }\end{array}$} & \multicolumn{3}{|c|}{ Años de experiencia } & \multicolumn{3}{|c|}{ Procedencia } \\
\hline & $\mathrm{N}$ & $\%$ & & $\mathrm{~N}$ & $\%$ & & N & $\%$ & & N & $\%$ & & $\mathrm{~N}$ & $\%$ \\
\hline Mujeres & 176 & 91,19 & $\begin{array}{c}20-25 \\
\text { años }\end{array}$ & 38 & 19,69 & Sí & 147 & 76,17 & Ninguno & 25 & 12,95 & $\begin{array}{l}\text { Comunidad } \\
\text { Valenciana }\end{array}$ & 137 & 70,98 \\
\hline \multirow[t]{13}{*}{ Hombres } & 17 & 8,81 & $\begin{array}{c}26-30 \\
\text { años }\end{array}$ & 49 & 25,39 & No & 46 & 23,83 & < 1 año & 29 & 15,03 & $\begin{array}{l}\text { Castilla y } \\
\text { León }\end{array}$ & 3 & 1,55 \\
\hline & & & $\begin{array}{l}+31 \\
\text { años }\end{array}$ & 106 & 54,92 & & & & $\begin{array}{l}1 \text { y } 3 \\
\text { años }\end{array}$ & 39 & 20,21 & Andalucía & 16 & 8,29 \\
\hline & & & & & & & & & + 4 años & 100 & 51,81 & Extremadura & 3 & 1,55 \\
\hline & & & & & & & & & & & & $\begin{array}{l}\text { Castilla y la } \\
\text { Mancha }\end{array}$ & 6 & 3,11 \\
\hline & & & & & & & & & & & & País Vasco & 4 & 2,07 \\
\hline & & & & & & & & & & & & Murcia & 1 & 0,52 \\
\hline & & & & & & & & & & & & Aragón & 5 & 2,59 \\
\hline & & & & & & & & & & & & Islas Canarias & 2 & 1,04 \\
\hline & & & & & & & & & & & & Madrid & 4 & 2,07 \\
\hline & & & & & & & & & & & & Cataluña & 6 & 3,11 \\
\hline & & & & & & & & & & & & La Rioja & 1 & 0,52 \\
\hline & & & & & & & & & & & & $\begin{array}{l}\text { Principado de } \\
\text { Asturias }\end{array}$ & 3 & 1,55 \\
\hline & & & & & & & & & & & & Galicia & 2 & 1,04 \\
\hline
\end{tabular}

\subsection{Procedimiento e instrumento de recogida de los datos}

Los participantes de este estudio estaban cursando la $2^{\text {a }}$ edición de un MOOC sobre "el aula inclusiva hoy en día" (octubre-noviembre, 2017). Durante el transcurso del mismo se proporcionó en la plataforma del curso el enlace de dicho cuestionario, diseñado mediante la herramienta de Google formulario. Los interesados podían participar de forma voluntaria, garantizando la confidencialidad de los datos.

El diseño metodológico que rige este estudio es de tipo descriptivo, se utiliza el método de encuesta y el cuestionario como instrumento (basado en Sans, 2004), el cual fue diseñado ad hoc. Este está formado principalmente por dos partes: 1) datos de tipo más contextual y demográfico (sexo, edad, trabajo en educación, 
experiencia docente, procedencia) y 2) preguntas acerca de la formación (p.ej.: ¿Te has formado o has leído sobre la observación con una lista de control o Check list?), el nivel de dominio (p.ej.: Nivel de conocimiento o dominio que crees tener sobre la observación con una lista de control o Check list) y puesta en práctica en el aula (p.ej.: ¿Has puesto en práctica en el aula la observación con una lista de control o Check list?). Las técnicas o métodos de evaluación sobre las cuales se ha preguntado han sido: check list, registro de incidentes críticos, diarios de aula, portafolio, evaluación de una presentación y defensa de un producto/resultado, entrevista de evaluación, evaluación de trabajos escritos, memorias e informes, cuestionarios KPSI, matrices o rúbricas de valoración, examen escrito (ensayo, preguntas cortas, test...), contrato didáctico o de aprendizaje, evaluación auténtica, evaluación formadora, evaluación entre iguales y autoevaluación.

Se ha combinado preguntas dicotómicas (Sí/No) con una escala tipo Likert de 1 a 5 ( 1 =No lo conozco/ no lo comprendo y $5=$ Lo domino de manera que sabría explicarlo). Se trata de un instrumento fiable, pues la prueba alfa de Cronbach arroja un valor de .927. El análisis de datos fue realizado mediante el SPSS Statistics 19. Asimismo, con dicho programa se calcularon las frecuencias, porcentajes, media y desviación típica y se elaboraron las tablas de resultados.

\section{RESULTADOS}

Los resultados se presentan siguiendo los objetivos planteados en la investigación.

Tal y como muestra la Tabla 2, el método de evaluación sobre el cuál más se han formado y han leído los docentes es la autoevaluación con un 91,7\%, seguido de los exámenes escritos (ensayo, preguntas cortas, test...) con un $88,6 \%$ y de las matrices o rúbricas de valoración con un $82,4 \%$. En el otro extremo, y por tanto los métodos o técnicas de evaluación sobre los que menos se han formado los participantes encontramos con un 19,7\% la evaluación auténtica. Finalmente resaltar que los cuestionarios KPSI son uno de los instrumentos de evaluación sobre los cuales menos se han formado y leído los participantes en este estudio con un porcentaje del $17,1 \%$. 
a) Formación o documentación que tienen los docentes sobre las diferentes técnicas y métodos de evaluación (dimensión del saber)

\section{TABLA 2}

\section{Formación o documentación sobre las diferentes técnicas y métodos de evaluación}

\begin{tabular}{rrr} 
& \multicolumn{2}{c}{ Sí } \\
\cline { 2 - 4 } & N & $\%$ \\
\hline lista de control o Check list & 96 & 49,7 \\
registro de incidentes críticos & 51 & 26,4 \\
diarios de aula & 152 & 78,8 \\
pvaluación de una presentación y defensa de un producto/resultado & 149 & 77,2 \\
entrevista de evaluación & 94 & 39,9 \\
evaluación de trabajos escritos, memorias e informes & 147 & 76,2 \\
cuestionarios KPSI (Knowledge and Prior Study Inventory) & 33 & 17,1 \\
matrices o rúbricas de valoración & 159 & 82,4 \\
examen escrito (ensayo, preguntas cortas, test, ...) & 171 & 88,6 \\
contrato didáctico o de aprendizaje & 86 & 44,6 \\
evaluación auténtica & 38 & 19,7 \\
evaluación formadora & 98 & 50,8 \\
evaluación entre iguales & 142 & 73,6 \\
autoevaluación & 177 & 91,7 \\
\hline
\end{tabular}

b) Grado de dominio de las diferentes técnicas y métodos de evaluación (dimensión del saber cómo)

En cuanto al nivel de dominio que creen tener los participantes (ver Tabla 3), vamos a detenernos a dos niveles de análisis': primero un análisis global y, a continuación, uno más específico.

Si nos fijamos en dónde creen tener mayor pericia los docentes (agregación de nivel 4 y 5) son los exámenes escritos $(46,7 \%)$, seguido de la autoevaluación $(38,4 \%)$ y las matrices de evaluación (30,1\%). En el extremo opuesto (nivel 1) encontramos que los cuestionarios KPSI $(76,7 \%)$ es el elemento donde menos dominio presenta el profesorado. Le sigue la evaluación auténtica $(73,1 \%)$ y el registro de incidentes críticos $(59,1 \%)$.

1 Dado que la desviación típica es elevada, la media no resulta un buen indicador, por lo que hemos optado por un análisis de frecuencias. 
TABLA 3

\section{Grado de dominio en relación a las diferentes técnicas y métodos de evaluación}

\begin{tabular}{|c|c|c|c|c|c|c|c|c|c|c|c|c|}
\hline & \multicolumn{2}{|c|}{$\begin{array}{l}\text { 1. No lo } \\
\text { conozcol } \\
\text { no lo } \\
\text { comprendo }\end{array}$} & \multicolumn{2}{|c|}{$\begin{array}{l}\text { 2. Lo } \\
\text { conozco } \\
\text { un poco/ lo } \\
\text { comprendo } \\
\text { un poco }\end{array}$} & \multicolumn{2}{|c|}{$\begin{array}{l}\text { 3. Lo } \\
\text { conozco } \\
\text { bastante/ lo } \\
\text { comprendo } \\
\text { bastante } \\
\end{array}$} & \multicolumn{2}{|c|}{$\begin{array}{l}\text { 4. Lo } \\
\text { conozco } \\
\text { bien/ lo } \\
\text { comprendo } \\
\text { bien }\end{array}$} & \multicolumn{2}{|c|}{$\begin{array}{l}\text { 5. Lo domino } \\
\text { de manera } \\
\text { que sabría } \\
\text { explicarlo a } \\
\text { otra persona }\end{array}$} & \multirow[b]{2}{*}{ M } & \multirow[b]{2}{*}{ DT } \\
\hline & $n$ & $\%$ & $n$ & $\%$ & $\mathrm{n}$ & $\%$ & $n$ & $\%$ & $n$ & $\%$ & & \\
\hline lista de control o Check list & 54 & 28,0 & 86 & 44,6 & 33 & 17,1 & 15 & 7,8 & 5 & 2,6 & 2,12 & 992 \\
\hline registro de incidentes críticos & 114 & 59,1 & 54 & 28,0 & 19 & 9,8 & 3 & 1,6 & 3 & 1,6 & 1,59 & .850 \\
\hline diarios de aula & 22 & 11,4 & 72 & 37,3 & 49 & 25,4 & 37 & 19,2 & 13 & 6,7 & 2,73 & 1.105 \\
\hline portafolio & 24 & 12,4 & 77 & 39,9 & 50 & 25,9 & 30 & 15,5 & 12 & 6,2 & 2,63 & 1.082 \\
\hline $\begin{array}{r}\text { evaluación de una } \\
\text { presentación y defensa de un } \\
\text { producto/resultado }\end{array}$ & 87 & 45,1 & 59 & 30,6 & 27 & 14,0 & 17 & 8,8 & 3 & 1,6 & 1,91 & 1.040 \\
\hline entrevista de evaluación & 77 & 39,9 & 65 & 33,7 & 32 & 16,6 & 16 & 8,3 & 3 & 1,6 & 1,98 & 1.020 \\
\hline $\begin{array}{r}\text { evaluación de trabajos } \\
\text { escritos, memorias e } \\
\text { informes }\end{array}$ & 26 & 13,5 & 62 & 32,1 & 65 & 33,7 & 27 & 14,0 & 13 & 6,7 & 2,68 & 1.084 \\
\hline $\begin{array}{r}\text { cuestionarios KPSI } \\
\text { (Knowledge and Prior Study } \\
\text { Inventory) }\end{array}$ & 148 & 76,7 & 32 & 16,6 & 10 & 5,2 & 3 & 1,6 & 0 & 0 & 1,32 & .644 \\
\hline $\begin{array}{r}\text { matrices o rúbricas de } \\
\text { valoración }\end{array}$ & 19 & 9,8 & 69 & 35,8 & 47 & 24,4 & 44 & 22,8 & 14 & 7,3 & 2,82 & 1.115 \\
\hline $\begin{array}{l}\text { examen escrito (ensayo, } \\
\text { preguntas cortas, test, ...) }\end{array}$ & 7 & 3,6 & 41 & 21,2 & 55 & 28,5 & 59 & 30,6 & 31 & 16,1 & 3,34 & 1.093 \\
\hline $\begin{array}{r}\text { contrato didáctico o de } \\
\text { aprendizaje }\end{array}$ & 88 & 45,6 & 53 & 27,5 & 22 & 11,4 & 23 & 11,9 & 7 & 3,6 & 2,01 & 1.175 \\
\hline evaluación auténtica & 141 & 73,1 & 30 & 15,5 & 10 & 5,2 & 8 & 4,1 & 4 & 2,1 & 1,47 & .924 \\
\hline evaluación formadora & 82 & 42,5 & 63 & 32,6 & 29 & 15,0 & 15 & 7,8 & 4 & 2,1 & 1,94 & 1.037 \\
\hline evaluación entre iguales & 38 & 19,7 & 73 & 37,8 & 37 & 19,2 & 33 & 17,1 & 12 & 6,2 & 2,52 & 1.168 \\
\hline autoevaluación & 9 & 4,7 & 49 & 25,4 & 61 & 31,6 & 47 & 24,4 & 27 & 14,0 & 3,18 & 1.104 \\
\hline
\end{tabular}

Si abordamos el análisis individual de cada uno de los métodos o técnicas mencionados en el párrafo anterior vemos que en cuanto al nivel de dominio que creen tener los encuestados sobre los exámenes escritos (ensayo, preguntas cortas, test...) detectamos como el 3,6\% no lo conocen o no lo comprenden, el $21,2 \%$ lo conocen o lo comprenden un poco, el 28,5\% lo conocen o lo comprenden bastante, un $30,6 \%$ lo conocen o lo comprenden bien y un $16,1 \%$ lo dominan. 
En cuanto a la autoevaluación cabe decir que solamente el 4,7\% de los participantes esgrimen que no la conocen o no la comprenden, el 25,4\% la conocen o la comprenden un poco, el 31,6\% la conocen o la comprenden bastante, el $24,4 \%$ la conocen o la comprenden bien y el $14,0 \%$ la dominan.

Por lo que respecta a las matrices o rúbricas de evaluación, percibimos como el $9,8 \%$ no lo conocen o no lo comprenden, el 35,8\% lo conocen o lo comprenden un poco, el 24,4\% lo conocen o lo comprenden bastante, el 22,8\% lo conocen o lo comprenden bien y el 7,3\% lo dominan.

El cuestionario KPSI no es comprendido ni conocido por el $76,7 \%$ de los encuestados, el $16,6 \%$ afirman que lo conocen o lo comprenden un poco, el 5,2\% lo conocen o lo comprenden bastante, el 1,6\% lo conocen o lo comprenden bien y nadie manifiesta dominar dicho instrumento de evaluación.

La evaluación auténtica no es conocida ni comprendida por el 73,1\% de los encuestados, el 15,5\% lo conocen o lo comprenden un poco, el 5,2\% lo conocen o lo comprenden bastante, el $4,1 \%$ lo conocen o lo comprenden bien y el 2,1\% lo dominan.

Finalmente, en cuanto al registro de incidentes críticos se aprecia como el 59,1\% no lo conocen o no lo comprenden, el $28,0 \%$ lo conocen o lo comprenden un poco, el 9,8\% lo conocen o lo comprenden bastante, el 1,6\% lo conocen o lo comprenden bien y el $1,6 \%$ lo dominan.

c) Puesta en práctica de las diferentes técnicas y métodos de evaluación en el aula (dimensión del hacer)

Tal y como se recoge en la Tabla 4, en referencia al uso o puesta en práctica de las diferentes técnicas y métodos de evaluación en el aula, los resultados arrojan que la autoevaluación es el método de evaluación más utilizado por el profesorado con un $60,1 \%$. Los exámenes escritos (ensayo, preguntas cortas, test...) con un 57,5\% es el segundo porcentaje más elevado en cuanto al uso de dicho instrumento de evaluación en el aula. En el otro extremo, hallamos con porcentajes similares, que un 11,4\% utilizan los registros de incidentes críticos y un 10,4\% utilizan la evaluación auténtica. Por último decir que apenas un 3,1\% afirman utilizar el cuestionario KPSI como instrumento de evaluación en sus aulas. 
TABLA 4

Uso de las diferentes técnicas y métodos de evaluación en el aula

\begin{tabular}{rcc}
\cline { 2 - 3 } & \multicolumn{2}{c}{ Sí } \\
\cline { 2 - 3 } & N & $\%$ \\
\hline lista de control o Check list & 46 & 23,8 \\
registro de incidentes críticos & 22 & 11,4 \\
diarios de aula & 83 & 43,0 \\
portafolio & 47 & 24,4 \\
evaluación de una presentación y defensa de un producto/resultado & 41 & 21,2 \\
enaluación de trabajos escritos, memorias e informes & 95 & 49,2 \\
cuestionarios KPSI (Knowledge and Prior Study Inventory) & 6 & 3,1 \\
matrices o rúbricas de valoración & 95 & 49,2 \\
examen escrito (ensayo, preguntas cortas, test, ...) & 111 & 57,5 \\
contrato didáctico o de aprendizaje & 39 & 20,2 \\
evaluación auténtica & 20 & 10,4 \\
evaluación formadora & 47 & 24,4 \\
evaluación entre iguales & 73 & 37,8 \\
autoevaluación & 116 & 60,1 \\
\hline
\end{tabular}

\section{DISCUSIÓN DE RESULTADOS Y CONCLUSIONES}

La competencia evaluativa es necesaria e imprescindible en el profesorado (Banco Mundial, 2007; Caena, 2014; Cano, 2005, FIER, 2010; Sarramona, 2007; OCDE, 2009; Perrenoud, 2004; UNESCO, 2015), máxime en un contexto de acelerados procesos de cambio y donde está adquiriendo protagonismo un currículum orientado al desarrollo de competencias del alumnado.

Los docentes deben conocer y saber aplicar un conjunto variado de instrumentos y técnicas de evaluación (Tejada, 2010) para conseguir recoger evidencias de aprendizaje lo más fiables y precisas posibles. Asimismo, deben garantizar otras condiciones como la complementariedad entre la evaluación sumativa y la formativa, la aplicación de pruebas en entornos lo más realistas posibles (Monereo, 2009), así como otorgar un mayor protagonismo a la autorregulación del alumnado, lo que nos hemos referido aquí como evaluación democrática (López, González y Barba, 2006; Murillo e Hidalgo, 2016). 
No obstante, tal y como hemos puesto de manifiesto, hay estudios (Elola y Toranzos, 2000; OCDE, 2005) que demuestran que los docentes tienen dificultades para cambiar sus prácticas en este campo y asocian la evaluación a exámenes, como tarea calificadora y con una fuerte presencia de la habilidad memorística, en detrimento de otras. Uno de los motivos que explica este problema reside en la falta de atención de la competencia evaluativa desde los programas de formación docente (Flórez, 1999; Martínez Rizo, 2013; Palacios y López-Pastor, 2011; Stiggins, 2007; Tejedor, 1998).

En este contexto, nos hemos planteado en la presente investigación analizar la competencia evaluativa de los docentes, con un objetivo triple y basándonos en los niveles de la propuesta de Miller (1990). El primer objetivo, pretendía averiguar si los docentes se han formado o han leído sobre diferentes técnicas y métodos de evaluación (saber). El segundo, conocer el nivel de dominio que tienen los docentes de las técnicas y métodos evaluativos (saber cómo) y el tercero, comprobar si han puesto en práctica en el aula dicha competencia evaluativa (hacer).

Los resultados obtenidos arrojan que en relación al objetivo uno (saber) y tres (hacer), la autoevaluación es el método sobre el cual más se han formado o leído y el más utilizado en el aula. Seguido de los exámenes escritos (ensayo, preguntas cortas, test...), tanto a nivel de saber cómo de hacer. Posteriormente, se puede decir que las matrices o rúbricas de valoración, los diarios de aula y portafolio son los instrumentos sobre los que también se han formado los participantes en porcentajes elevados. No obstante, si nos centramos en el eje de hacer, después de los exámenes; la evaluación de trabajos escritos, memorias e informes y las matrices o rúbricas de valoración son unos de los más puestos en práctica en el aula. Continuando con el uso, el método de evaluación entre iguales pese a que los participantes se han documentado o han leídos sobre este método, su implementación en el aula es menor. Lo mismo ocurre con los diarios de aula y portafolio. De los resultados obtenidos se puede decir que en relación a la evaluación formadora, la lista de control, la entrevista de evaluación, el contrato dialógico o de aprendizaje y la presentación y defensa de un producto/resultado, los participantes afirman formarse o leer más sobre dichas técnicas o métodos de evaluación que ponerlas en práctica en sus aulas (todas con porcentajes inferiores). Subrayar que los registros de incidentes críticos, la evaluación auténtica y los cuestionarios KPSI son los instrumentos o métodos de evaluación que menos conocen (saber) y utilizan (hacer) en el aula. 
Pasando al segundo objetivo de nuestro estudio (relativo al saber cómo), el examen escrito (ensayo, preguntas cortas, test...) y la autoevaluación son los instrumentos o métodos de evaluación que más competentes se perciben los encuestados. Sin embargo, los cuestionarios KPSI, los registros de incidentes críticos y la evaluación auténtica son los que menor grado de dominio presentan.

En cuanto a las limitaciones del estudio es necesario comentar que, aunque, la metodología utilizada nos ha permitido arrojar un poco de luz en cuanto a los objetivos que nos habíamos marcado con esta investigación, conviene mencionar que el instrumento que hemos utilizado no se trata de un instrumento validado, no obstante ha demostrado tener un alto grado de fiabilidad. Además, aunque partíamos de la propuesta de Sans (2004) para el diseño del cuestionario, también se hubiese podido preguntar en el instrumento de recogida de los datos por otros métodos de evaluación, como por ejemplo la evaluación sumativa o formativa. Son aspectos que, sin duda, se podrían considerar e incluir en estudios posteriores. En cuanto al tamaño de la muestra decir que es reducida pero de utilidad para este tipo de estudios preliminares, tal y como afirma Morales (2017).

En cualquier caso, queremos poner de manifiesto a través de esta investigación que es momento que el profesorado se abra hacia una nueva cultura evaluadora, superando las barreras al cambio. La competencia de evaluación debe servir, además, para algo más que acreditar, en un contexto tan cambiante como el que nos encontramos y donde cobra cada vez más fuerza la dinámica de aprender a aprender y el desarrollo de competencias del alumnado. En este contexto, la diferenciación de los procedimientos de evaluación y el uso de técnicas orientadas a la evaluación continua y formativa (Tejada, 2010), se manifiesta como necesario para enriquecer y mejorar los aprendizajes, que además suelen ser muy valoradas por los propios estudiantes (Bain, 2007).

Asumir los axiomas mencionados en el campo de la evaluación no es en sí revolucionario, sino que se trata de una nueva lógica, una nueva "mirada". En la línea de lo que plantea Cano (2008):

Pasemos de una evaluación de los aprendizajes a una evaluación para los aprendizajes y busquemos que ésta logre el impacto último que cualquier reforma educativa debiera buscar: que nuestros alumnos aprendan mejor y estén más preparados para afrontar el futuro (p.14). 
Para hacer posible este avance en la mentalidad del docente tenemos que seguir investigando en la competencia autopercibida de la evaluación, adecuar los currículos formativos y, especialmente, mejorar la formación inicial del profesorado, tanto en los agentes responsables de la formación como en la metodología. En relación al segundo aspecto, diversas investigaciones demuestran la importancia de emplear herramientas de autoevaluación docente, que permitan poner en marcha una práctica reflexiva que ayude a educadores a analizar cómo pueden mejorar sus prácticas evaluativas. Dado que sin reflexión, no hay cambio y la reflexión permite la mejora del aprendizaje de las competencias docentes (Brookfield, 1995; Gómez, 2011).

\section{BIBLIOGRAFÍA}

Arellano, M., Jara, R., Merino, C., Quintanilla, M. y Cuéllar, L. (2008). Estudio comparativo de dos instrumentos de evaluación diagnóstica aplicados a profesores de Química en formación: un estudio piloto. Revista Electrónica de Enseñanza de las Ciencias, 7 (1), 1-22. Disponible en: http://reec.uvigo.es/volumenes/volumen7/ ART1 Vol7 N1.pdf

Bain, K. (2007). Lo que hacen los mejores profesores universitarios. Valencia: Universidad de Valencia.

Banco Mundial (2007). Ampliar oportunidades y construir competencias para los jóvenes: Una agenda para la educación secundaria. Colombia: Banco Mundial-Mayol Ediciones.

Bernal, J.L. y Teixidó, J. (2009). Las competencias docentes en la formación del profesorado. Madrid: Síntesis.

Bisquerra, R. (2007). Metodología de la investigación educativa. Madrid: La Muralla.

Brookfield, S. (1995). Becoming a Critically Reflective Teacher. San Francisco, CA: Jossey Bass.

Caena, F. (2014). Teacher competence frameworks in Europe: policy-as-discourse and policy-as-competence. European Journal of Education, 49 (3), 311-331. doi: 10.1111/ejed.12088

Cano, E. (2005). Cómo mejorar las competencias de los docentes: Guia para la autoevaluación y el desarrollo de las competencias del profesorado. Barcelona: Graó.

Cano, E. (2008). La evaluación por competencias en la educación superior. Revista de currículum y formación del profesorado, 12 (3), 1-16.

Cohen, L., Manion, L. y Morrison, K. (2000). Research Methods in Education (6th edition). London, Routledge Falmer.

Cohen, R. y Swerdlik, M. (2009). Psychological Testing and Assessment: An Introduction to Test and Measurement (7th Edition). Boston: McGraw-Hill Higher Education 
Covacevich, C. (2014), Cómo seleccionar un instrumento para evaluar aprendizajes estudiantiles. Banco Interamerciano de Desarrollo, 1-40. Disponible en: $\underline{\text { https:// }}$ publications.iadb.org/bitstream/handle/11319/6758/Cómo-seleccionar-uninstrumento-para-evaluar-aprendizajes-estudiantiles.pdf

Darr, C., 2005. A Hitchhiker's Guide to Validity. Disponible en: http://assessment.tki.org.nz/ Assessment-tools-resources/Assessment-tool-selector

De Miguel, M. (2005). Modalidades de enseñanza centradas en el desarrollo de competencias. Orientaciones para promover el cambio metodológico en el EEES. Madrid: Ministerio de Educación-Universidad de Oviedo.

Elola, N. y Toranzos, L. V. (2000). Evaluación Educativa: una aproximación conceptual. Recuperado de http://bibliotecadigital.academia.cl/bitstream/handle/1234567 89/585/?sequence $=1$

Fernández March, A.(2006). Metodologías activas para la formación de competencias. Educatio siglo XXI, 24 35-56.

FIER (2010). Education and Training 2010: Three studies to support School Policy Development. Lot 2: Teacher Education Curricula in the EU. Final Report. Jyväskylä: University of Jyväskylä. Disponible en: $\underline{\text { http://ktl.jyu.fi/img/portal/17545/ }}$ TEC FINAL REPORT 12th Apr2010 WEB.pdf

Flórez, J. (1999). Evaluación de la calidad de la docencia. León: Universidad de León.

Fortea, M.A., Sánchez-Tarazaga, L. y Zorrilla-Silvestre, L. (2017). Aprendizaje en entornos laborales y evaluación auténtica. @TIC, Revista d'innovació educativa, 19, 17-21.

Galvis, R. (2007). De un perfil docente tradicional a un perfil docente basado en competencias. Acción pedagógica, 16, 48-57.

Gómez, M.V. (2011). Desarrollo profesional del maestro. La competencia reflexiva. Tesis doctoral. Universitat de Lleida, Lleida.

Jiménez, V. y Naranjo, M. (2014). Educational assessment of cooperative learning in the Program CL/LC: cooperating to learn, learning to cooperate. ECER The Past, the Present and the Future of Educational Research EUROPEAN EDUCATIONAL RESEARCH ASSOCIATION

Jorba, J. y Sanmartí, N. (1996). Enseñar, aprender y evaluar: un proceso de regulación continua. Madrid: MEC.

López, V.M. (2005). La participación del alumnado en la evaluación: la autoevaluación, la coevaluación y la evaluación compartida. Tándem. 17

López, V.M., González, M. y Barba, J.J. (2006). ¿Debe el alumnado participar en la evaluación? Propuestas y experiencias en Primaria y Secundaria. Recuperado de http://www. concejoeducativo. org/article.php?id article $=89$

Martínez Rizo, F. (2013). Dificultades para implementar la evaluación formativa. Revisión de literatura. Perfiles educativos, 35 (139), 128-150. Disponible en http://www.scielo.org.mx/scielo.php?script=sci arttextgpid $=$ S0185-26982013000100009 
Martínez-Rojas, J. G. (2008). Las rúbricas en la evaluación escolar: su construcción y su uso. Avances en Medición, 6, 129-138

Massot, I., Dorio, I. y Sabariego, M. (2012). Estrategias de recogida y análisis de la información. En R. Bisquerra (coord.), Metodología de la investigación educativa (pp. 329366). Madrid: La Muralla.

Miller, G.E. (1990). The assessment of clinical skills/competence/performance. Acad Med, 65(9), 63-67.

Monereo, C. (coord.) (2009). Pisa como excusa. Repensar la evaluación para cambiar la enseñanza. Barcelona: Graó.

Monereo, C., Sànchez-Busqués, S. y Suñé, N. (2012). La enseñanza auténtica de competencias profesionales. Un proyecto de aprendizaje recíproco instituto-universidad, Revista de currículum y formación del profesorado, 16, 1, 79-101. Disponible en: http:// www.ugr.es/ recfpro/rev161ART6.pdf

Morales, P. (2009). Ser profesor: una mirada al alumno. Guatemala: Universidad Rafael Landivar.

Morales, P. (2017). Tamaño necesario de la muestra. ¿Cuántos sujetos necesitamos? Universidad Pontificia de Comillas, 1-87. Disponible en: http://www2.df.gob. mx/virtual/evaluadf/docs/gral/taller2015/S0202EAC.pdf

Murillo, F. J. e Hidalgo, N. (2015). Dime cómo evalúas y te diré qué sociedad construyes. Revista Iberoamericana de Evaluación educativa, 8(1), 5-9. Disponible en

Murillo, F.J. e Hidalgo, N. (2016). Editorial. Evaluación Democrática y para la Democracia. Revista Iberoamericana de Evaluación Educativa, 9(1), 5-7.

Naranjo, M. y Jiménez, V. (2015). La evaluación del aprendizaje cooperativo: un reto abordable. En Mayordomo y Onrubia (Coords.). El aprendizaje cooperativo. Barcelona, España: Editorial UOC.

OCDE (2005). Formative Assessment. Improving learning in secondary classrooms. París: OCDE. Disponible en: http://www.oecd.org/education/ceri/35661078.pdf

OCDE (2009). Los docentes son importantes: Atraer, formar y conservar a los docentes eficientes. París: OCDE.

Palacios, A. y López-Pastor, V. M. (2011). Haz lo que yo digo pero no lo que yo hago: sistemas de evaluación del alumnado en la formación inicial del profesorado. Revista de Educación, 361, 279-305.

Perrenoud, P. (2004). Diez nuevas competencias para enseñar. Barcelona: Graó.

Prats, E. (2016). La formación inicial docente entre profesionalismo y vías alternativas: mirada internacional. Bordón, 68(2), 19-33. doi:10.13042/Bordon.2016.68201

Sánchez-Tarazaga, L. (2017). Las competencias docentes del profesorado de Educación Secundaria: Importancia percibida e implicaciones en la formación inicial. Tesis doctoral, Castellón de la Plana. 
Sanmartí, N. (2007). 10 ideas clave: Evaluar para aprender. Barcelona: Graó.

Sanmartí, N. (2010). Avaluar per aprendre. L'avaluació per millorar els aprenentatges de I'alumnat en el marc del currículum per competències. Recuperado de http://xtec. gencat.cat/web/.content/alfresco/d/d/workspace/SpacesStore/0024/fc53024f626e-423b-877a-932148c56075/avaluar per aprendre.pdf

Sanmartí, N. y Alimenti, G. (1998). La evaluación refleja el modelo didáctico: análisis de actividad de evaluación planteadas en clases de química. Educación Química, 15 (2), 60-68.

Sans, A. (2004). L'avaluació dels Aprenentatges. Construcció d'instruments (Núm. 2). ICE-UB. Disponible en: http://www.ub.edu/ice/node/57

Sarramona, J. (2007). Las competencias profesionales del profesorado de secundaria. Estudios sobre Educación, 12, 31-40.

Stiggins, R.J.(2007). Conquering the Formative Assessment Frontier. En James H. McMillan (ed.), Formative Classroom Assessment: Theory into practice (pp.8-27). Nueva York: Teachers College Press.

Tamir, P. y Lunetta, V.M.(1978). An Analyst of laboratory activities in the BSCS. Yellow version, American Biology Teacher, 40, 426-428.

Tejada, J. (2010). La evaluación de las competencias en contextos no formales: dispositivos e instrumentos de evaluación. Revista de Educación, 354,731-745.

Tejedor, F. J. (Dir.). (1998). Las estrategias utilizadas por los profesores universitarios para la evaluación del aprendizaje de los alumnos. Madrid: CIDE

Tejedor, F.J. y García-Valcárcel, A. (2010). Evaluación del desempeño docente. Revista española de Pedagogía, 247, 439-459.

Tiana, A. (2013). Los cambios recientes en la formación inicial del profesorado en España: una reforma incompleta. Revista Española de Educación Comparada, 22 (2), 39-58.

Trillo Alonso, F. (2005). Competencias docentes y evaluación auténtica: ¿Falla el protagonista? Colección de Cuadernillos de actualización para pensar la Enseñanza Universitaria, 3, 1-20. Recuperado de http://www.unrc.edu.ar/unrc/academica/ pdf/cuadernillo03.pdf

UNESCO (2015). Replantear la educación: ¿Hacia un bien común mundial? París: UNESCO. 
